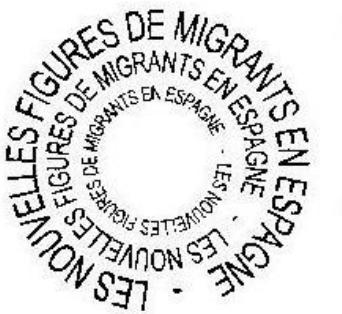

\title{
ÉVOLUTION DES MIGRATIONS ROUMAINES EN ESPAGNE : VERS UNE CIRCULATION RURALE ?
}

\author{
Vincent MAISONGRANDE *
}

Actuellement, les migrations roumaines ont pris une importance de tout premier plan dans le très contrasté paysage migratoire contemporain de l'Espagne. Timidement arrivés dans le pays au tout début des années 1990, le nombre de Roumains a crû progressivement, de manière de plus en plus accélérée, jusqu'à devenir de nos jours la principale nationalité étrangère en nombre. Selon les chiffres de 2008 , ils étaient plus de $730000^{1}$, c'est-à-dire plus nombreux que les Marocains ou les Équatoriens, traditionnellement majoritaires en Espagne. Lorsque l'on compare ce chiffre avec celui de 2000 - 6410 Roumains recensés - la progression semble hors du commun en moins d'une décennie.

Par rapport à la thématique générale des migrations internationales, de même qu'au regard des logiques de mobilité des Roumains vers l'espace espagnol et au sein de celui-ci, les conséquences d'une telle croissance en volume sont évidemment très importantes. Que ce soit au niveau des directions successives prises par ces flux de populations, des dispositifs migratoires sur lesquels ils reposent ou encore pour ce qui est des structures sociales internes aux groupes migrants et qui sous-tendent leurs mobilités, nous verrons que les évolutions ont été nombreuses ef tout à fait symptomatiques.

Pourtant, il nous semble difficile de pleinement comprendre les mouvements qui secouent actuellement ce champ migratoire roumain en Espagne sans les replacer préalablement au sein du cadre plus large qui est le leur. Il s'agit des contextes d'origine de ces mouvements en Roumanie même, mais aussi et surtout de l'historique des mobilités de ces populations à travers toute l'Europe depuis le début des années 1990. C'est dans ce double cadre, que nous évoquerons au long du texte, qu'il convient d'analyser les phénomènes migratoires roumains contemporains

\footnotetext{
* Sociologue, géographe, MIGRINTER, CNRS - Université de Poitiers.

1. INSTITUTO NACIONAL DE ESTADÍSTICA, Padrón de habitantes, 2008, Espagne.
} 
en Espagne, dont les évolutions les plus récentes ne constituent à nos yeux qu'un développement somme toute assez logique et s'inscrivant tout à fait dans la continuité des phénomènes analogues observés en d'autres lieux pour ces mêmes populations migrantes.

Tout au long de notre contribution nous tenterons de mettre l'accent à la fois sur l'aspect social et spatial de ces mobilités, les deux étant intimement liés tant du point de vue théorique qu'empirique. C'est cette réciprocité qui nous permet, en observant les déplacements, de comprendre les structures sociales sur lesquelles ils s'appuient, et, en observant le fonctionnement des réseaux migratoires roumains, de saisir la mise en place par leurs membres de telles ou telles formes de mobilité. Nous insisterons ainsi sur une notion qui nous semble centrale et éclairante, que l'on verra apparaître de manière continue dans différents contextes : celle de captation des ressources et d'accès à différentes occasions socialement et spatialement circonscrites.

Précisons enfin que nous nous appuierons très largement sur les travaux conceptuels et empiriques de différents auteurs, mais aussi sur nos propres travaux d'observation et de recueil de récits de parcours migratoires et d'expériences, travaux réalisés dans le cadre d'une recherche préalable auprès d'une famille précise de migrants roumains, elle-même intégrée à des groupes et des réseaux circulatoires et migratoires plus étendus ayant développé des mobilités internationales nombreuses et répétées durant près de 20 ans dans un grand nombre de pays européens. Ces observations et recueils ont justement été effectués dans l'optique d'une compréhension globale et plurifactorielle fine du phénomène et nous permettront d'en offrir une illustration éclairante ${ }^{2}$.

2. La mise en place de l'échantillon étudié résulte d'un travail d'observation participante débuté en 2005 auprès de groupes de migrants roumains originaires de la province de Mehedinti au sudouest de la Roumanie. Des entretiens biographiques complets ont été réalisés auprès de 11 individus préalablement sélectionnés suite à cette longue période d'observation comme présentant des profils et des historiques migratoires diversifiés, parfois particulièrement représentatifs des expériences de l'ensemble du groupe, parfois particulièrement atypiques. Tous les individus interrogés appartiennent à la même famille élargie (dans le sens où ils entretiennent entre eux des liens de parenté à divers degrés) et à trois générations successives. Le groupe familial ainsi constitué, et principalement organisé pour ce qui est de la migration autour d'une dizaine de familles nucléaires, regroupe au total plus d'une soixantaine de personnes. Il s'agit là du cœur de notre échantillon auprès duquel les recueils de parcours ont été les plus systématiques. Par ailleurs, l'ensemble de l'échantillon concerné par nos observations et incluant cette famille mais aussi tous les différents contacts sociaux plus ou moins éloignés dont elle dispose représente plus de 400 personnes, dans leur majorité originaires de la même région de Roumanie, et liées entre elles par des liens familiaux, de voisinage, de travail ou de simple connaissance. Ce groupe est constitué comme un grand réseau social plus ou moins intégré et caractérisé par sa participation commune, à différents niveaux, et décliné en différents rôles et stratégies, mais selon des logiques analogues, aux phénomènes de mobilités internationales que nous allons décrire. Le choix de nous centrer sur cette famille précise est lié à des considérations méthodologiques, non seulement parce qu'elle constitue un très bon exemple des pratiques et logiques migratoires à 


\section{Contextes ef logiques d'origine des migrations roumaines}

À partir de 1989, le principal fait social en Roumanie est la très forte "crise économique" que connaît le pays, non résolue à ce jour. Suite au choc brutal qu'a représenté la chute du régime de Ceaușescu — qui a mis une bonne partie de la population au chômage - et comme dans beaucoup d'autres anciens "pays communistes", la progressive inclusion du pays dans l'économie de marché se traduira par une fulgurante croissance des disparités économiques qui verra quelques familles s'enrichir énormément tandis que la grande majorité s'enfoncera peu à peu dans une misère vertigineuse et surtout durable.

En réponse à cette "nouvelle donne" et pour éviter la relégation, la solution rapidement et de plus en plus largement adoptée par certains Roumains et certaines familles roumaines sera le recours à des stratégies de cumul d'activités : travail temporaire ou par périodes, commerce sur le marché parallèle, agriculture de subsistance, récupération, troc et "coups de main" divers... ${ }^{3}$ Pour notre échantillon comme pour de nombreux Roumains, ces stratégies se baseront sur la recherche et la captation de toutes les possibilités et ressources disponibles, en jouant sur les contacts sociaux et les appartenances diverses (locales, ethniques...), les expériences, les compétences et le savoir-faire de tout un chacun, mais aussi de plus en plus sur la mobilité. L'idée sera de cumuler des revenus variés afin d'assurer une subsistance devenue impossible par le seul travail salarié. À mesure que l'inclusion sur le marché mondial se fera plus complète, on verra généralement le niveau de vie des familles baisser car les prix s'aligneront sur les normes européennes tandis que les salaires resteront très faibles (concurrence internationale oblige), et ces logiques se généraliseront au sein de la société roumaine ${ }^{4}$. Les populations concernées développeront rapidement une très forte capacité d'adaptation et de "débrouille" basée sur un mode d'organisation en réseaux sociaux de plus en plus larges et parfois construits progressivement.

l'œuvre dans l'ensemble du groupe, mais également parce qu'elle nous a permis d'obtenir une vision très fine, et jusqu'au niveau interpersonnel, des rapports sociaux pouvant avoir une influence sur les mobilités.

3. Cf. DUCHÊNE, Gérard, "Les revenus informels en Roumanie : estimation par enquête", Revue d'Études comparatives Est-Ouest, vol. 4, n 106, 1999, pp. 35-64; MOROKVASIC, Mirjana, "La mobilité transnationale comme ressource : le cas des migrants d'Europe de l'Est", Cultures \& Conflits, $\mathrm{n}^{\circ} 33-34$, printemps-été 1999, pp. 105-122; POTOT, Swanie, Vivre à l'Est, travailler à l'Ouest: les routes roumaines de l'Europe, Paris : Éd. L'Harmattan, 2007, $226 \mathrm{p}$.

4. On estime aujourd'hui que, en Roumanie, l'émigration concernerait entre 3 et 4 millions de personnes pour une population totale de 22 millions d'habitants. 
Au sein de cet ensemble d'occasions diverses, l'émigration temporaire prendra une place croissante, car de plus en plus facile et rentable à mesure que les frontières européennes s'ouvriront aux Roumains et que leurs réseaux sociaux s'étendront à l'étranger (par adaptation à ces nouvelles conditions), gagnant en amplitude et en efficacité à l'heure de favoriser l'expatriation d'un nombre toujours plus important de personnes. Cette maturation progressive des dispositifs migratoires entrainera une évolution dans le temps des logiques, des pratiques et des destinations de la migration, évolution que nous pourrons clairement voir à l'œuvre dans le cas de l'Espagne, mais aussi ailleurs en Europe, et que nous détaillerons ici en trois périodes historiques.

Notons enfin que dans le cas des Roumains ces transformations successives s'inscrivent dans une évolution "de fond" des migrations internationales issue de la combinaison à la fois des possibilités croissantes de mobilité (transports et communications) des migrants mais aussi de politiques toujours plus restrictives en matière d'entrée et de séjour dans les pays de destination. Cette situation paradoxale entraine l'adoption de stratégies de mobilité visant à compenser la précarité de l'accès à l'emploi et la mise en place de combinaisons d'activités de plus en plus atypiques, que l'on peut aujourd'hui observer pour un nombre croissant de migrants à travers le monde 5 .

\section{Flux migratoires "ethniques" et premières circulations en Europe : le rôle prépondérant des réseaux sociaux}

Au cours de la première période, outre le développement des logiques que nous avons évoqué en Roumanie même, dès la chute du régime de Ceaușescu en 1989 on verra aussi se mettre en place deux types de mouvements vers l'étranger différenciés mais liés entre eux. D'une part, des flux de réfugiés fuyant la Roumanie et des groupes ethniques ou religieux minoritaires qui quitteront le pays, s'inscrivant ainsi dans les reconfigurations nationales et spatiales générales à cette époque dans toute l'Europe de l'Est ${ }^{6}$; d'autre part, apparaîtront des pratiques de

5. Cf. TARRIUS, Alain, "Territoires circulatoires et espaces urbains. Différenciation des groupes migrants", in : MOROKVASIC, Mirjana ; RUDOLPH, Hedwig (sous la direction de), Migrants : les nouvelles mobilités en Europe, Paris : Éd. L'Harmattan, 1996, pp. 93-117; PORTES, Alejandro, "La mondialisation par le bas: l'émergence des communautés transnationales", Actes de la Recherche en Sciences Sociales, $n^{\circ} 129$, septembre 1999, pp. 15-25; PERALDI, Michel, Cabas et containers : activités marchandes informelles et réseaux migrants transfrontaliers, Paris : Éd. Maisonneuve \& Larose, 2001, $361 \mathrm{p}$.

6. Qu'il s'agisse de regroupement (les deux Allemagnes) ou de séparation (Yougoslavie, Tchécoslovaquie), mais aussi de transferts de populations minoritaires entre différents pays. 
va-et-vient et de circulations migratoires tout d'abord limitées mais régulières et qui seront souvent le fait de populations frontalières ou en lien avec celles étant déjà parties. Ainsi s'appuieront-elles sur les premiers flux de réfugiés par le biais de contacts sociaux préexistants entre groupes, qui seront alors mis à contribution à dessein afin de pouvoir accéder à de nouvelles possibilités de travail ou de commerce à l'étranger. En ce sens, les principales destinations pour les deux types de flux seront alors les mêmes et auront des liens avec les nombreuses minorités existant en Roumanie - Allemands, Hongrois, Serbes ou encore Turcs et juifs - même si de petits groupes se rendront bien plus loin, dans des logiques là encore exploratoires ${ }^{7}$.

Les parcours de la famille que nous avons suivie illustrent bien cette première période de mobilités circulatoires basée sur une instrumentalisation stratégique des appartenances. Ses membres commenceront tout d'abord par utiliser leurs liens avec des populations roms de leur zone d'origine pour pouvoir se rendre avec elles en Allemagne et s'y faire passer pour des réfugiés (les gains venant des allocations accordées par l'État allemand dans l'attente d'une décision relative à la demande d'asile). Par la suite, ils s'intégreront dans l'activité du commerce de détail en Roumanie et multiplieront les navettes et les travaux ponctuels entre leur région frontalière, la Serbie et la Bulgarie, s'appuyant pour ce faire sur les contacts variés dont ils pourront disposer et qu'ils pourront se créer sur les marchés.

C'est selon de telles logiques que les premiers migrants roumains arriveront en Espagne au début des années 1990. Deux principaux groupes seront repérés dès cette époque dans la région de Madrid : une communauté de protestants adventistes originaires de Teleorman qui s'implantera à Coslada, une autre arrivée depuis Alba lulia en Transylvanie et qui après un bref passage en Suède s'installera à Alcalá de Henares. Ces deux premiers groupes seront constitués autour de pionniers arrivés seuls et un peu par hasard en Espagne, lesquels auront rapidement tendance à regrouper autour d'eux leurs familles, voisins et amis, selon une logique de migration en réseaux.

7. Cf. DIMINESCU, Dana, "Faire une saison. Pour une anthropologie des migrations roumaines en France. Le cas du pays d'Oas", Migration Études, n 91 , novembre-décembre 1999, 16 p. ; MICHALON, Bénédicte, "Circuler entre Roumanie et Allemagne. Les Saxons de Transylvanie, de l'émigration ethnique au va-et-vient", in : Congrès Études Balkaniques : état des savoirs et pistes de recherche, Paris, 2002 ; REY, Violette, "Les Roumains sur les chemins de l'Europe", in: DIMINESCU, Dana (sous la direction de), Visibles mais peu nombreux : les circulations migratoires roumaines, Paris : Éd. de la Maison des Sciences de l'Homme, 2003, pp. 27-32. 
L'arrivée de ces migrants en Espagne se fera alors irrégulièrement et beaucoup y déposeront des demandes d'asile pas toujours justifiées pour pouvoir y rester, comme cela se faisait dans d'autres pays. Rapidement pourtant cette pratique sera abandonnée, car ils se rendront compte qu'ils peuvent s'y implanter de manière irrégulière et s'intégrer dans le vaste marché du travail parallèle. Leurs principales zones d'implantation - liées aux possibilités locales et au hasard des espaces des premières arrivées - seront Madrid et sa banlieve, Zaragoza et les villes de la côte Est, en particulier Valence et Castellón. Les hommes s'intégreront largement dans le secteur du bâtiment, les femmes dans celui des services domestiques ${ }^{8}$.

Si ces communautés resteront au départ limitées à quelques familles, elles pourront surtout jouer le rôle de "tête de pont" migratoire, offrant (ou monnayant) leur aide et leur expérience à d'autres Roumains venus de tout le pays, leur permettant ainsi de s'inscrire dans les mêmes activités et les mêmes espaces en Espagne ${ }^{9}$.

À partir de 1994, tous les pays européens s'orienteront vers un rejet des demandes d'asile déposées par des Roumains, mais les flux de migrants économiques vont se maintenir tant bien que mal. On assistera surtout à une diversification des destinations et des pratiques dans le cadre d'explorations circulatoires dont le rayon s'accroîtra progressivement. Les Roumains se feront alors plus nombreux en France, en Italie, en Grande-Bretagne et en Espagne, chaque destination impliquant, par adaptation, des stratégies de passage particulières et, sur place, des activités spécifiques. Les obstacles juridiques à l'immigration étant de plus en plus nombreux dans tous ces pays, ces flux devront se baser toujours plus sur les groupes de Roumains déjà implantés et sur les dispositifs (de transport et d'entraide) qui se perfectionneront peu à peu.

Ainsi, avec l'entrée de l'Espagne dans l'espace Schengen en 1992, il sera possible pour les migrants économiques et les demandeurs d'asile présents dans le reste de l'Europe de l'Ouest, attirés par les possibilités de travail qu'offre le pays, de s'y rendre plus facilement, ce qu'ils feront surtout après que l'Allemagne (1993) et la France (1998) auront durci leur politique d'immigration à l'égard des Roumains. Ceux qui vien-

8. Cf. DOMINGO, Andreu; GIL, Fernando; MAISONGRANDE, Vincent, "La inserción laboral de los inmigrantes rumanos y búlgaros en España", Cuadernos de Geografía, n 84, 2008, pp. 213-236.

9. Aujourd'hui encore, les principaux espaces de concentration des populations roumaines sont ceux que nous avons cités, de même que leurs principaux secteurs d'activité. 
dront directement depuis la Roumanie devront d'abord obtenir des visas de tourisme.

Dans tous les cas, il leur faudra généralement s'appuyer sur les réseaux sociaux déjà implantés localement. L'obtention de visas, par exemple, sera conditionnée à la présentation d'une invitation préalablement établie par un national ou un résident étranger en situation régulière. Les pionniers roumains qui avaient pu être régularisés en $1991^{10}$ vont commencer à en établir, parfois contre rémunération ${ }^{11}$. II en va de même pour ce qui est du travail, puisque l'on sait que les contacts sociaux sont capitaux pour pouvoir intégrer les "niches" réservées aux immigrés en Espagne ${ }^{12}$. Pour trouver un logement ou obtenir une éventuelle régularisation par le travail, il s'agira également de passer par les contacts que les pionniers avaient pu établir avec les populations locales et par l'intermédiation de ces pionniers qui ne sera pas toujours gratuite, mais qui pour les nouveaux arrivants constituera la manière la plus efficace de réussir leur migration ${ }^{13}$.

Au sein des réseaux migratoires ainsi créés on constatera l'apparition de positions de pouvoir différenciées et de structures internes signifiantes, puisque le traitement et l'aide apportée ne seront pas les mêmes pour un membre direct de la famille d'un pionnier que pour une vague connaissance rencontrée en Roumanie ou en Espagne même.

Dans l'ensemble pourtant, la situation des Roumains s'améliorera progressivement. Ils seront plutôt bien accueillis par la population et les entrepreneurs qui favoriseront leur implantation et leur intégration écono-

10. En 1991, à l'occasion de la première procédure de régularisation massive qui concernera 146502 personnes, dont certains Roumains arrivés avant cette date.

11. On verra à cette époque en Roumanie même l'apparition de nombreuses agences de "contrats en origine", mandatées par des entreprises espagnoles cherchant de la main-d'œuvre étrangère et offrant des contrats de travail temporaires assortis d'un visa. En fait, ces contrats seront généralement "vendus" au plus offrant. Voir PAJARES, Miguel, "La red social en los procesos de inserción laboral de los inmigrantes rumanos", $V^{\circ}$ Congreso nacional sobre la inmigración en España, Valencia, 2007

12. Cf. DE MIGUEL LUKEN, Verónica ; SOLANA SOLANA, Miguel, Redes sociales de apoyo: la inserción de la población extranjera, Bilbao : Fundación BBVA, 2007, 320 p.

13. II s'agira souvent de l'encaissement de diverses commissions par les pionniers. Les migrants roumains, tout à fait conscients de ces pratiques, devront généralement s'y conformer en attendant de maîtriser la langue et de créer leur propre réseau de contacts au sein de la population locale et de pouvoir se transformer à leur tour en "tête de pont" migratoire pour leur famille et leurs amis. Voir BLEAHU, Ana, "Romanian migration to Spain. Motivation, networks and strategies", in : POP, Daniel (Ed.), New patterns of labour migration in CEE, Cluj-Napoca: AMM Design, 2004, pp. 21-35 ; PAJARES, Miguel, Inmigrantes del Este. Procesos migratorios de los rumanos, Barcelona : Editorial Icaria, 2007, $274 \mathrm{p}$ 
mique ${ }^{14}$. L'Espagne deviendra ainsi une destination de plus en plus prisée tout au long des années 1990.

\section{Après 2002 : croissance des flux, déstabilisation des réseaux, évolution des mobilités}

La seconde période débute en 2002 avec l'accès des ressortissants roumains à la libre circulation touristique dans l'ensemble de l'espace Schengen et la mise en place de visas temporaires dont l'obtention est simplifiée. Ce changement, allié au rôle désormais central des réseaux sociaux - il n'y a plus d'explorations au hasard ou de pionniers qui partent seuls - provoque une rapide explosion des flux migratoires roumains, mais aussi une transformation de leurs caractéristiques jusque-là observées. Ces flux se polarisent vers l'Europe occidentale, et des populations de plus en plus jeunes et de moins en moins préparées se lancent à leur tour dans l'aventure migratoire ${ }^{15}$.

Pour le groupe que nous avons suivi, c'est alors la France, où les membres disposeront préalablement de contacts éloignés, qui constituera leur principale destination. Les logiques quills y rencontreront et leurs évolutions seront pourtant les mêmes que celles que l'on pourra observer en Espagne, mais leurs activités seront différentes, basées surtout sur la mendicité, le travail illégal étant bien plus difficile à exercer en France ${ }^{16}$.

En Espagne, comme ailleurs en Europe, la croissance du nombre de migrants roumains est donc fulgurante, le phénomène étant surtout observé dans les zones principales d'implantation des communautés roumaines (Madrid, Zaragoza et Castellón) qui se trouvent rapidement saturées, ce qui entraîne diverses conséquences négatives : le nombre de Roumains qui demandent de l'aide aux pionniers augmente fortement et ces derniers ne peuvent plus suivre, les logements pour les immigrés viennent à manquer ${ }^{17}$

14. Et surtout par rapport à d'autres populations immigrées encore plus marginalisées comme les Marocains que les Roumains seront amenés à substituer progressivement dans certains espaces et pour accomplir certains travaux. Voir POTOT, Swanie, "Mobilités en Europe. Étude de deux réseaux migratoires roumains", Sociologie Românească, serie noua, $n^{\circ} 2,2000$, pp. 97-115; MATA ROMEU, Anna, "La lógica empresarial entorno a la contratación de trabajadores inmigrantes", $V^{\circ}$ Congreso nacional sobre la inmigración en España, Valencia, 2007.

15. Cf. MARCU, Sylvia, "Dinámica y estructura migratoria laboral de los rumanos : 1990-2006. Flujos de emigración hacia Espan̄a, destino Madrid", Migraciones, n²1, junio 2007, pp. 115-157.

16. Cf. MORICE, Alain, "Trafics de main-d'œuvre et emploi illégal : les irréguliers dans l'étau des textes et des pratiques", Hommes \& Migrations, n 1214, juillet-août 1998, pp. 28-37.

17. Car il s'agit, tout comme pour les emplois accessibles aux immigrés, d'un marché fortement
segmenté. 
et l'offre de main-d'œurre dépassant la demande, les salaires "au noir" ainsi que l'activité de tous va baisser.

Si auparavant les réseaux disposaient d'un certain contrôle sur les arrivées car en fait c'étaient eux qui répartissaient les visas et les offres de travail, ils vont être totalement déstabilisés par l'ampleur du phénomène suite au changement juridique. La logique de regroupement qui jusqu'à présent était bénéfique pour tous va déboucher sur l'apparition de phénomènes de concurrence entre les Roumains pour la captation des possibilités disponibles au niveau local.

En retour, les réseaux migratoires vont se contracter, c'est-à-dire que les pionniers ne vont plus offrir leur aide aussi largement et auront tendance à se concentrer sur leur famille la plus proche. Les contacts établis avec les nationaux pouvant offrir travail et papiers ne se partageront plus autant et les logiques de méfiance, de contrôle social et d'exploitation au sein des groupes se feront encore plus fortes.

Face à cette dégradation objective ef rapide de la situation des Roumains dans ces espaces, on assistera à la mise en place de nouvelles vagues de mobilités exploratoires et concentriques au sein même de l'Espagne. À la recherche de nouvelles possibilités de travail, les individus et les familles se dirigeront de plus en plus largement vers le sud du pays et les espaces ruraux, où ils pourront souvent s'installer et établir leurs propres contacts au niveau local, et où ils s'intégreront en plus grand nombre dans l'activité agricole. Les Roumains vont ainsi se disperser à partir de quelques foyers, et ils commenceront à être nombreux, voire majoritaires parmi les immigrés dans un nombre croissant de provinces espagnoles. Les arrivées postérieures se feront d'ailleurs de plus en plus directement dans ces zones rurales, sans passer par les régions d'implantation "traditionnelles"

On voit ici encore un exemple de la manière dont peut fonctionner l'articulation entre l'organisation sociale interne des migrants et leur mobilité spatiale, puisqu'une déstabilisation de l'un des termes de la relation entraîne une évolution de l'autre. Ces phénomènes s'appuient sur l'application de stratégies d'adaptation rapide et se basent sur une capacité à naviguer entre des espaces physiques et sociaux variés à la re-

18. Cf. VIRUELA MARTÍNEZ, Rafael, "Inmigrantes rumanos en España: aspectos territoriales y procesos de sustitución laboral", Scripta Nova. Revista Electrónica de Geografía y Ciencias Sociales, vol. $10, n^{\circ} 222,2006,26$ p. ; MAISONGRANDE, Vincent, Les mobilités des migrants: implantations et dispersions comparées en Espagne, mémoire de diplôme universitaire SIGmage (Systèmes d'information géographique et méthodes d'analyse géographiques), Département de géographie, Université de Poitiers, 2008, 72 p. 
cherche de nouvelles ressources mobilisables. II s'agit de logiques que l'on avait pu observer en Roumanie dès le départ, mais qui seront par la suite appliquées dans différents pays et contextes à l'étranger. Les réseaux sociaux des migrants roumains en Espagne, et pour autant les possibilités qu'ils pourront offrir, auront dès lors tendance à être plus étendus et diversifiés, mais en revanche les liens en leur sein perdront en intensité et les ressources auxquelles ils permettront l'accès seront globalement plus précaires.

$\mathrm{Ce}$ que nous apprennent les parcours des migrants dont nous avons recueilli le récit en France, c'est que des schémas analogues ef liés aux mêmes causes se répéteront à cette même époque pour le même type de population partout en Europe. Arrivés en 2003 dans des zones de forte concentration de Roumains telles que Nantes ou La Rochelle, ils verront très vite leur nombre croître et leur activité (la mendicité) s'essouffler, et ils devront commencer à s'enfoncer à l'intérieur des terres et à se déplacer sans cesse d'une ville à une autre pour continuer de pratiquer leur activité avec succès, dans des espaces non encore saturés de Roumains. Ces stratégies de recours à la mobilité circulatoire s'avéreront très efficaces, mais pendant un temps seulement, car elles finiront par s'ébruiter et les nouveaux espaces seront saturés à leur tour. Les Roumains que nous avons suivis se dirigeront alors en grand nombre vers l'Espagne ${ }^{19}$. Par ailleurs, cela permet de constater le rôle que jouent les réseaux migratoires dans la reproduction des mêmes activités dans les mêmes espaces de destination.

Cette logique type des migrations roumaines que nous voyons ici apparaître en filigrane, basée sur la séquence exploration-imitationconcentration-saturation-nouvelle dispersion, est bien celle qui nous permet aujourd'hui de comprendre les évolutions observables de ces mouvements.

\section{Les mobilités actuelles : vers une recrudescence des cir- culations?}

Ce que nous souhaiterions mettre en lumière pour la troisième période, la plus récente, c'est que, puisque ce phénomène de croissance des flux migratoires roumains et de saturation consécutive se répétait dans toute

19. Cf. MAISONGRANDE, Vincent, Les circulations migratoires roumaines en Europe : réseaux sociaux et inscription dans l'espace, mémoire de master II en migrations internationales, MIGRINTER, Université de Poitiers, 2008, $161 \mathrm{p}$. 
l'Europe, entrainant une baisse des gains de tous et partout ${ }^{20}$, à une échelle globale on a assisté à une réorientation vers les pays qui apparaissaient comme les plus accueillants en termes de travail et de possibilités d'installation : l'ltalie et surtout l'Espagne. Ainsi, à partir de 2004-2005, le nombre de Roumains en Espagne continue de croître encore plus fortement, cette fois-ci dans tout le pays, et les nouvelles zones d'implantation sont rapidement saturées à leur tour, d'autant plus qu'il s'agissait de zones rurales n'offrant que peu d'activités stables. La situation y devenant alors critique - comme cela avait pu se produire peu auparavant en France - on assiste encore à des adaptations des stratégies migratoires et de mobilité plutôt que de se résigner à voir baisser la rentabilité globale de cette destination.

Sur ce point nous nous appuyons plus précisément encore sur le cas du groupe étudié qui l'illustre parfaitement. Son entrée en Espagne avait eu lieu par le biais de deux pionniers arrivés en 2002 et qui s'étaient rendus directement dans une zone rurale, l'un à Alcanar, dans le sud de la Catalogne (province de Tarragone), l'autre à Almonte, en Andalousie (province de Huelva). Ces pionniers ont travaillé principalement dans l'agriculture, mais ont aussi réussi à s'intégrer dans d'autres activités qu'ils ont pu alterner tout au long de l'année, pouvant ainsi s'installer durablement. Assez vite ils ont aussi pu établir des contacts avec les entrepreneurs locaux et favoriser l'implantation autour d'eux de petites communautés roumaines. Selon les logiques évoquées précédemment, ils se sont bien gardés d'aider trop de monde et ont réservé les meilleures occasions pour leur famille la plus proche, n'hésitant pas à exploiter les autres (cela dépend tout de même des cas, les logiques d'exploitation dès le départ étant bien plus fortes en Andalousie, le pionnier en Catalogne étant apparemment "plus honnête").

Rapidement pourtant, la présence roumaine dans ces espaces croîtra fortement à son tour (voir tableau 1), et surtout du fait de réorientations depuis la France et l'Italie de membres des familles déjà implantées, l'activité et les salaires de l'ensemble des travailleurs en situation irrégulière baisseront notablement et de plus en plus à partir de $2004^{21}$.

20. Lorsqu'il ne s'agit pas d'un fort rejet de la part des populations locales, comme ce sera le cas en Italie à partir de 2007.

21. Tim, arrivé à Alcanar en 2004 , nous expliquera au cours d'un entretien que les salaires "au noir" dans l'agriculture pouvaient être alors de $50 €$ par jour, alors qu'en 2008 ils ne seront plus que de $25 €$ ou $30 €$, la logique de concurrence étant que vu le nombre de Roumains présents sur place, "si cela ne te convient pas, le patron ira chercher quelqu'un d'autre qui sera prêt à accepter ce prix». 
Tableau 1 : Évolution de la présence roumaine dans les communes citées et à leurs alentours entre 2004 et 2008

\begin{tabular}{|c|c|c|c|c|c|c|c|c|}
\hline \multirow[b]{2}{*}{ Commune } & \multicolumn{5}{|c|}{ Roumains } & \multirow{2}{*}{$\begin{array}{c}\begin{array}{l}\text { Population } \\
\text { totale }\end{array} \\
2008\end{array}$} & \multirow{2}{*}{$\begin{array}{r}\% \text { Roumains dans } \\
\text { la population totale } \\
2008\end{array}$} & \multirow{2}{*}{$\begin{array}{c}\begin{array}{c}\text { Progression } \\
\text { Roumains }\end{array} \\
2004-2008\end{array}$} \\
\hline & 2004 & 2005 & 2006 & 2007 & 2008 & & & \\
\hline Alangary & 415 & 583 & 792 & 977 & 1281 & 10510 & 12,19 & $209 \%$ \\
\hline 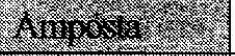 & 201 & 345 & 488 & 684 & 1025 & 20652 & 4,96 & $410 \%$ \\
\hline $\begin{array}{l}\text { Sani Carles de } \\
\text { ta Repitat }\end{array}$ & 493 & 709 & 779 & 1003 & 1436 & 15307 & 9,38 & $191 \%$ \\
\hline 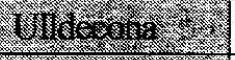 & 455 & 606 & 589 & 885 & 1162 & 6987 & 16,63 & $155 \%$ \\
\hline Bencarto 3 & 283 & 412 & 591 & 806 & 1096 & 26381 & 4,15 & $287 \%$ \\
\hline Joraross & 261 & 444 & 640 & 903 & 1182 & 27912 & 4,23 & $353 \%$ \\
\hline Peniscola & 61 & 135 & 191 & 321 & 456 & 7560 & 6,03 & $648 \%$ \\
\hline Almonte & 115 & 387 & 767 & 1057 & 1727 & 21452 & 8,05 & $1402 \%$ \\
\hline Hinojos & 3 & 13 & 80 & 96 & 91 & 3807 & 2,39 & $2933 \%$ \\
\hline Pilas & 28 & 50 & 149 & 289 & 491 & 12988 & 3,78 & $1654 \%$ \\
\hline $\begin{array}{l}\text { Villamanrique } \\
\text { de la Condesa }\end{array}$ & 17 & 48 & 133 & 153 & 229 & 4097 & 5,59 & $1247 \%$ \\
\hline
\end{tabular}

Source : Padrón municipal de habitantes. Instituto Nacional de Estadística. Les niveaux de gris indiquent les zones de rattachement de chaque commune.

Dans ce contexte, l'implantation durable de nouveaux arrivants roumains sera très difficile et moins rentable, l'aide des pionniers de plus en plus rare et intéressée; quant au travail, il deviendra de plus en plus temporaire et précaire. Et, selon ce qui a été indiqué, ce cas de figure semble se répéter dans d'autres espaces ruraux du pays ${ }^{22}$ et dans lesquels les membres de la famille disposent de contacts sociaux mais trop éloignés pour leur permettre l'accès à des offres de travail fixe, les plus recherchées.

Nous avons ici tous les éléments préalables au développement de pratiques circulatoires en Espagne qui pourront justement s'appuyer sur le "quadrillage" de l'espace par les réseaux sociaux roumains. Puisque les possibilités de travail sont plus diversifiées (activités et localisation spatiale) mais aussi généralement plus précaires (et dans le cas de l'agriculture souvent temporaires), on voit certains Roumains et certaines familles roumaines qui commencent à se déplacer constamment entre diverses

22. Différentes villes agricoles de taille moyenne situées en Andalousie, Estrémadure, Castilla-La Mancha et dans la région de Valence. 
régions du pays, alternant différents travaux de récolte mais aussi des emplois temporaires dans le tourisme à différentes époques de l'année, cumulant ainsi les activités afin de maintenir leurs gains en situation de migration. Pour ce faire, ils utiliseront leur longue expérience de la "débrouille" et de la circulation acquise depuis des années en Roumanie et en Europe, mais surtout les nombreux contacts "faibles" dont ils pourront disposer au sein des réseaux migratoires, réseaux sociaux créés au départ en Roumanie mais qui au cours des années et des vagues d'émigration temporaires successives se seront dispersés et étoffés.

À partir de 2007, avec l'accès des Roumains au droit de libre circulation dans toute l'Europe, ces circulations en Espagne (devenu leur principal pays de destination) pourront alterner avec des périodes d'activité temporaires dans d'autres pays, en France, en Italie, en Grande-Bretagne ou en Grèce, là encore basées sur les contacts créés au fil des années et des réorientations. On aura ainsi des formes d'unification ponctuelles des champs circulatoires à l'échelle de tout le continent.

Il convient tout de même de modérer ce constat, car si les contacts, les expériences et les aides pourront se diffuser à de larges populations au sein des réseaux, ceux-ci disposeront tout de même de structures internes importantes et signifiantes par rapport aux mobilités. Ainsi, en fonction des stratégies familiales ou individuelles propres, des compétences (professionnelles ou linguistiques) de tout un chacun et des contacts plus ou moins proches au sein du réseau social global, chaque sous-groupe disposera tout de même de ses destinations et de ses activités de prédilection, mais qui pourront toujours s'articuler le cas échéant avec des périodes de travail, de commerce ou de mendicité dans un autre pays ou une autre région, et ce bien sûr avec de fréquents retours dans leur zone d'origine en Roumanie.

\section{En guise de conclusion}

Cette rapide rétrospective historique nous permet d'appréhender les formes modernes de mobilités migratoires roumaines qui se mettent en place à l'heure actuelle en Espagne et en Europe. II s'agit pourtant de phénomènes très difficiles à appréhender à une échelle globale et qui restent pratiquement invisibles, si ce n'est en passant par l'étude fine et sur le long terme de populations et de groupes concrets. Certains indices statistiques nous permettent pourtant de penser que ces pratiques tendent à se généraliser toujours plus en Espagne : la dispersion croissante des po- 
pulations roumaines ou leur taux de variation interprovinciale anormalement élevé ${ }^{23}$. Dans la présente contribution nous avons surtout cherché à mettre en lumière la construction progressive de ces circulations migratoires et la forte réciprocité qui existe entre mobilités et structures sociales des groupes migrants. C'est cette articulation qui, dans le cas des Roumains, explique la répétition de logiques analogues dans des espaces et à des époques très diverses, ce qui nous permet de penser qu'il s'agit là d'une "constante migratoire" propre à ces populations.

Quant à l'actuelle crise de l'emploi en Espagne, les Roumains figurent parmi les migrants ayant le plus largement opéré des retours dans leur pays d'origine. Ainsi, nombreux sont ceux qui, se retrouvant au chômage depuis le début de l'année 2009, ont décidé de repartir en Roumanie, mais surtout, aux dires mêmes des intéressés, pour y vivre à moindre frais le temps que dureront leurs allocations, en attendant de pouvoir revenir en Espagne dès que l'emploi sera relancé. Tous les Roumains n'agissent bien sûr pas de la sorte, mais seulement ceux qui se trouvaient dans le pays depuis un certain temps et qui avaient un emploi fixe leur ayant permis de cotiser le nombre de mois suffisant (un an minimum de travail déclaré pour l'Espagne) pour toucher leurs allocations. II n'y a pourtant pas de raison pour qu'il en aille de même pour les "circulants" et les migrants les plus précaires.

Ainsi, dans les espaces ruraux et malgré la dureté de la crise, l'afflux de travailleurs roumains continue de s'observer invariablement pendant les périodes de récolte, même si l'activité y est encore plus morcelée et temporaire, et les salaires encore plus faibles. Ne disposant de toute manière pas d'autres occasions de gains aussi stables et élevés ni en Roumanie ni ailleurs en Europe (la France, la Grande-Bretagne, l'Allemagne et l'Italie ne leur ayant pas accordé comme l'Espagne le droit au travail en 2009), il semble probable que les Roumains continueront de se rendre en Espagne, mais que la précarisation additionnelle que représente la "crise" pourra même y renforcer les logiques de pluriactivité et de circulation que l'on y observe déjà.

23. Leur taux de dispersion spatiale est en très forte croissance, bien plus que celui de tout autre nationalité immigrée dans le pays, de même que les déplacements migratoires de Roumains, internes à l'Espagne, de province à province. Voir MAISONGRANDE, Vincent, "La movilidad de los rumanos en España : análisis espacial y estadística a nivel de municipios", XII Congreso de inmigración, Almería, 2009 ; VIRUELA MARTíNEZ, Rafael, "Población rumana y búlgara en España : evolución, distribución geográfica y flujos migratorios", Cuadernos de Geografía, n ${ }^{\circ} 84$, 2008, pp. 164-194. 
Nombre de archivo: Documentol

Directorio:

Plantilla: $\quad$ C: $\backslash$ Users $\backslash$ Vincent y

Mira $\backslash$ AppData $\backslash$ Roaming $\backslash$ Microsoft $\backslash$ Plantillas $\backslash$ Normal.dot

Título:

Asunto:

Autor: Vincent y Mira

Palabras clave:

Comentarios:

Fecha de creación: $\quad$ 12/07/2011 13:55:00

Cambio número: 1

Guardado el:

Guardado por:

Tiempo de edición: 0 minutos

Impreso el: $\quad$ 12/07/2011 13:59:00

Última impresión completa

Número de páginas: $\quad 14$

Número de palabras: 4 (aprox.)

Número de caracteres: 24 (aprox.) 